\title{
Driving change: A partnership study protocol using shared emergency department data to reduce alcohol- related harm
}

\author{
Peter MILLER, ${ }^{1}$ Nicolas DROSTE ${ }^{0},{ }^{1}$ Diana EGERTON-WARBURTON, ${ }^{2}$ David CALDICOTT, ${ }^{3}$ \\ Gordian FULDE, ${ }^{4}$ Nadine EZARD, ${ }^{4}$ Paul PREISZ, ${ }^{4}$ Andrew WALBY, ${ }^{5}$ Martyn LLOYD-JONES, ${ }^{5}$ \\ Julian STELLA,${ }^{6}$ Michael SHERIDAN, ${ }^{6}$ Tim BAKER $\mathbb{D}^{7},{ }^{7}$ Michael HALL, ${ }^{8}$ Anthony SHAKESHAFT, ${ }^{9}$ \\ Alys HAVARD, ${ }^{10}$ Steve BOWE, ${ }^{1}$ Petra K STAIGER, ${ }^{1}$ Catherine D'ESTE, ${ }^{11,12}$ Chris DORAN, ${ }^{13}$ \\ Kerri COOMBER, ${ }^{1}$ Shannon HYDER, ${ }^{1}$ Daniel BARKER ${ }^{13}$ and Jonathan SHEPHERD ${ }^{14}$ \\ ${ }^{1}$ School of Psychology, Deakin University, Geelong, Victoria, Australia, ${ }^{2}$ School of Clinical Sciences at Monash Health, Monash University, \\ Melbourne, Victoria, Australia, ${ }^{3}$ Calvary Health Care, Canberra, Australian Capital Territory, Australia, ${ }^{4}$ St Vincent's Hospital Sydney, Sydney, New \\ South Wales, Australia, ${ }^{5}$ St Vincent's Hospital Melbourne, Melbourne, Victoria, Australia, ${ }^{6}$ Barwon Health, Geelong, Victoria, Australia, \\ ${ }^{7}$ Southwest Health Care, Warrnambool, Victoria, Australia, ${ }^{8}$ The Canberra Hospital and Health Service, Canberra, Australian Capital Territory, \\ Australia, ${ }^{9}$ National Drug and Alcohol Research Centre, The University of New South Wales, Sydney, New South Wales, Australia, ${ }^{10}$ Centre for \\ Big Data Research in Health, The University of New South Wales, Sydney, New South Wales, Australia, ${ }^{11}$ National Centre for Epidemiology and \\ Population Health, The Australian National University, Canberra, Australian Capital Territory, Australia, ${ }^{12}$ The University of Newcastle, Newcastle, \\ New South Wales, Australia, ${ }^{13}$ Central Queensland University, Rockhampton, Queensland, Australia, and ${ }^{14}$ Crime and Security Research \\ Institute, Cardiff University, Cardiff, UK
}

\begin{abstract}
Background: Sharing anonymised ED data with community agencies to reduce alcohol-related injury and assaults has been found effective in the UK. This protocol document outlines the design of an Australian multi-site trial using shared, anonymised ED data to reduce alcoholrelated harm.
\end{abstract}

Design and Method: Nine hospitals will participate in a 36 month stepped-wedge cluster randomised trial. After a 9 month baseline period, EDs will be randomised in five groups, clustered on geographic proximity, to commence the intervention at 3 monthly intervals. 'Lastdrinks' data regarding alcohol use in the preceding $12 \mathrm{~h}$, typical alcohol consumption amount, and location

Correspondence: Dr Nicolas Droste, School of Psychology, Deakin University Waterfront Campus, Level 3/27 Brougham Street, Geelong, VIC 3220, Australia. Email: nic. droste@deakin.edu.au

Peter Miller, PhD, Professor of Violence Prevention; Nicolas Droste, PhD, Research Fellow; Diana Egerton-Warburton, MBBS, FACEM, MClinEpi, MPH, CCPU, Director of Emergency Medicine Research; David Caldicott, MBBS, FRCEM, Emergency Consultant, Clinical Senior Lecturer; Gordian Fulde, MBBS, Director of Emergency Department; Nadine Ezard, MBBS, PhD, Clinical Director of Alcohol and Drug Service; Paul Preisz, MBBS, FACEM, Acting Director of Emergency Department; Andrew Walby, MBBS, Director of Emergency Medicine; Martyn Lloyd-Jones, MBChB, MRCGP, FRACGP, FAChAM, Director of Addiction Medicine; Julian Stella, MBBS, Head of Research and Co-convenor of Medical Students; Michael Sheridan, MBBS, Clinical Senior Lecturer, Emergency Director; Tim Baker, MBBS, MA, Director of Centre Rural Emergency Medicine; Michael Hall, MBBS, Staff Specialist, Clinical Lecturer; Anthony Shakeshaft, MA, PhD, Professor of Medicine; Alys Havard, MA, PhD, Senior Research Fellow; Steve Bowe, PhD, Senior Research Fellow Biostatistics; Petra K Staiger, PhD, Associate Professor; Catherine D'Este, PhD, Chair in Biostatistics; Chris Doran, PhD, Research Professor; Kerri Coomber, PhD, Research Fellow; Shannon Hyder, PhD, Lecturer; Daniel Barker, $\mathrm{PhD}$, Lecturer; Jonathan Shepherd, CBE, FMedSci, DDSc, PhD, FDSRCS, FRCS, FRCPsych, FRCEM, FFPH, FLSW, Emeritus Professor.

Accepted 5 February 2019

of alcohol purchase and consumption, are to be prospectively collected by ED triage nurses and clinicians at all nine EDs as a part of standard clinical process. Brief information flyers will be delivered to all ED patients who self-report risky alcohol consumption. Public Health Interventions to be conducted are: (i) information sharing with venues (via letter), and (ii) with police and other community agencies, and (iii) the option for public release of 'Top 5' venue lists.

Outcomes: Primary outcomes will be: (i) the number and proportion of ED attendances among patients reporting recent alcohol use; and (ii) the number and proportion of ED attendances during high-alcohol hours (Friday and Saturday nights, 20.00-06.00 hours) assigned an injury diagnosis. Process measures will assess logistical and feasibility concerns, and clinical impacts of implementing this systems-change model in an Australian context. An economic cost-benefit analysis will evaluate the economic impact, or return on investment.

Key words: alcohol, assault, emergency department, injury, public health intervention, violence. 


\section{Introduction}

This study protocol outlines the design of a multi-site trial evaluating the impact of sharing ED data on alcohol-related harm within the Australian context. The World Health Organization promotes the distribution and usage of ED data as a major component in public health approaches to prevent injuries. ${ }^{1,2} \mathrm{An}$ emerging trend is the use of ED recorded data on assaults and alcohol-related injuries as a tool for targeting police and other regulatory community level interventions (e.g. 'TASC' - Tackling Alcohol-related Street Crime). ${ }^{3}$ The method of anonymised ED data sharing to reduce injury, violence, and assaults has been frequently referred to as the 'Cardiff model'. In a systematic review of evidence for the model, ${ }^{4}$ seven of eight included studies were conducted in UK EDs. All studies that attempted to measure intervention effectiveness reported substantial reductions of assaults and ED attendances post-intervention, with the exception of one, which reported no change. ${ }^{5-12}$ In Cardiff, UK, police-recorded assault rates fell from seven to five a month per 100000 population compared with an increase from five to eight in comparison cities. ${ }^{8}$ Over a 6 year post data-sharing period in Wirral, UK, ED attendances for intentional injuries decreased by $35.6 \%$ and alcohol-related assault attendances decreased by $30.3 \% .^{5}$ Economic evaluations estimate that the cumulative social benefit is $£ 82$ GBP for each pound spent on the programme, with a cost-benefit ratio of 14.8 for the health service and 19.1 for the criminal justice system. ${ }^{13}$

Intervention trials of the Cardiff data-sharing model have yet to be implemented in Australia. The public health interventions in this study are based on sharing of anonymised data collected through 'last drinks' questions asked of all ED patients in participating hospitals. A pilot project conducted in a single hospital in regional Australia demonstrated the feasibility of collecting these data, which indicate sources and locations of alcohol-related harm. ${ }^{14}$ During this 6 month pilot trial, $10.8 \%$ of injury patients reported consuming alcohol in the $12 \mathrm{~h}$ prior to injury. During high-alcohol-hours (HAH; 20.00 hours Friday to 06.00 hours Saturday and 20.00 hours Saturday to 06.00 hours Sunday), alcoholrelated injuries accounted for $36.1 \%$ of all ED injury presentations. In total, $41.7 \%$ of alcohol-related attendances during HAH were among patients who reported consuming last drinks at identifiable hotels, bars, nightclubs or restaurants, or identifiable public areas/events. Approximately two-thirds $(60.2 \%)$ of attendances with alcohol-related presentations had purchased their alcohol at packaged liquor outlets. The pilot demonstrated the feasibility of implementing sustainable 'lastdrinks' data collection methods in the ED with minimal cost to the hospital, very high acceptance and cooperation rate among triage staff, and no impact to the quality of patient care. $^{14}$

Based on the success of the pilot study, we are undertaking an Australian multi-site stepped-wedge randomised clustered trial evaluating the impact of sharing anonymised ED data on alcohol-related harm. This protocol paper outlines the design of the trial, which has been funded by the National Health and Medical Research Council (NHMRC) Partnership Project scheme and St Vincent's Hospitals Australia with additional self-funding support provided by The Canberra Hospital, ACT Health.

\section{Method \\ Interventions}

Three public health data sharing interventions are based on the approaches previously trialled and reported in the UK by Shepherd et al. ${ }^{4}$ Similar avenues exist in Australia for public health interventions around alcohol as exist in the UK, such as dissemination of intelligence through state and city level police and liquor licensing bodies, and established systems of liquor accords and violent venues registers. Data can be also be used to inform and strengthen opposition to liquor expansions and developments, and provides important additional source of information for licensing regulators.

\section{Public health interventions}

All sites will engage in intervention methods (i) and (ii), with the option to opt out of intervention (iii) at the discretion of local partner investigators.

(i) Quarterly letter to licensed venues outlining how many ED presentations were among individuals who had been drinking in their venue prior to attendance.

In this intervention, the research team generate customised letters for the top five venues reported at each ED, outlining the number of attendances related to their business, anonymised details of the cases including prognoses and, where appropriate, anonymised photos of injuries. Letters will be delivered via the Australasian College for Emergency Medicine (ACEM) to registered licensees.

(ii) Anonymised quarterly data sharing with local authorities.

In intervention (ii), de-identified, aggregated data is prepared and shared with local police, licensing authorities and local government, identifying the top five venues reported in the relevant ED and providing an aggregated summary of alcohol-related attendances to the ED.

(iii) Quarterly public reports ranking the top five venues reported in the ED and giving anonymous case studies of the associated short- and long-term harms with excessive alcohol consumption.

Intervention (iii) will publicly report aggregated data every 3 months for each of the selected hospitals via press release from ACEM, about the source of alcohol for patients with alcohol-related presentations and the number of cases of 'last drinks' attendances for each local venue. Research support staff employed at each hospital will work with clinical staff to generate brief anonymised case studies to accompany data releases. Press releases will be publicly available for download via the ACEM website, will be 


\begin{tabular}{|l|c|c|c|c|c|c|c|c|c|c|c|c|}
\hline & $\mathbf{T}_{\mathbf{0}}$ & $\mathbf{T}_{\mathbf{1}}$ & $\mathbf{T}_{\mathbf{2}}$ & $\mathbf{T}_{\mathbf{3}}$ & $\mathbf{T}_{\mathbf{4}}$ & $\mathbf{T}_{\mathbf{5}}$ & $\mathbf{T}_{\mathbf{6}}$ & $\mathbf{T}_{\mathbf{7}}$ & $\mathbf{T}_{\mathbf{8}}$ & $\mathbf{T}_{\mathbf{9}}$ & $\mathbf{T}_{10}$ & $\mathbf{T}_{10}$ \\
\hline Cluster & $3 \mathrm{~m}$ & $6 \mathrm{~m}$ & $9 \mathrm{~m}$ & $12 \mathrm{~m}$ & $15 \mathrm{~m}$ & $18 \mathrm{~m}$ & $21 \mathrm{~m}$ & $24 \mathrm{~m}$ & $27 \mathrm{~m}$ & $30 \mathrm{~m}$ & $33 \mathrm{~m}$ & $36 \mathrm{~m}$ \\
\hline A (2 EDs) & 0 & 0 & 0 & 0 & 1 & 1 & 1 & 1 & 1 & 1 & 1 & 1 \\
\hline B (3 EDs) & 0 & 0 & 0 & 0 & 0 & 1 & 1 & 1 & 1 & 1 & 1 & 1 \\
\hline C (1 ED) & 0 & 0 & 0 & 0 & 0 & 0 & 1 & 1 & 1 & 1 & 1 & 1 \\
\hline D (1 ED) & 0 & 0 & 0 & 0 & 0 & 0 & 0 & 1 & 1 & 1 & 1 & 1 \\
\hline E (2 EDs) & 0 & 0 & 0 & 0 & 0 & 0 & 0 & 0 & 1 & 1 & 1 & 1 \\
\hline
\end{tabular}

Figure 1. Stepped-wedge cluster randomised trial design for nine EDs grouped into five geographic clusters. $0=$ control; $1=$ intervention active (all sites perform intervention (i) and (ii), and can opt-in to (iii). Unshaded (1) areas are the periods in which intervention is active. Grey-shaded (0) areas represent the control period where baseline data are collected. Geographic clustering: (A) Southwest Victoria, 2 EDs; (B) eastern suburban Melbourne, 3 EDs; (C) Central Melbourne, 1 ED; (D) Central Sydney, 1 ED; and (E) Canberra, 2 EDs.

distributed to local and state media outlets per site, and publicised via ACEM social media channels such as Twitter. ACEM Public Affairs staff will monitor media coverage and estimated audience demographics and exposure for each release for the duration of the project.

\section{Patient brief information pamphlet}

During project development, clinical partners highlighted the servicedelivery importance of including an immediate, patient-focused information handout in response to the information obtained through the data collection model. In an effort to proactively reduce drain on resources, many EDs utilise the presentation of alcohol-related injury and harm as an opportunity to motivate reduction in the patients' alcohol consumption and subsequent risk behaviours. ${ }^{15}$

There are mixed findings from reviews, trials and meta-analyses assessing the effectiveness of brief EDbased interventions at reducing risky alcohol consumption and harms, with conclusions varying according to the type and outcome measures. One systematic review reported no impact of single session intervention upon alcohol consumption, ${ }^{16}$ and an alternate meta-analysis also concluded that brief interventions based in the ED had no significant effect on consumption but had a small effect in reduced self-reported incidence of injury at patient follow up. ${ }^{17}$ As such, the inclusion of a clinical information handout in the current model meets a clinical obligation and strikes the best balance of evidence, cost, and clinical demand, but is not expected to result in significant changes in the outcome measures.

Double-sided A4 information handouts will be provided to all attendances who self-report risky alcohol consumption above the NHMRC guideline of two standard drinks on any day, ${ }^{18}$ and will be delivered during control and intervention phases of the project. Handouts contain structured advice for reducing alcohol consumption, Australian population drinking norms, and referral information for local services, in a format adapted from the Simple Structured Advice Intervention tool. ${ }^{15}$

\section{Design}

A stepped-wedge design will be used, with the duration of the trial being 36 months (Fig. 1). The steppedwedge cluster randomised trial is a form of cross-over design with unidirectional cross-over (from control to intervention). In the application of this design, the public health intervention will be introduced in a staggered roll-out where the sequence of clusters (i.e. EDs) and time-period is randomly allocated at the start of the trial. ${ }^{19-21}$ All clusters sequentially cross-over into an interventiondelivery phase in a randomly selected order, until all EDs are delivering the intervention (Fig. 1). The steppedwedge design was deemed most appropriate because the intervention model has demonstrable effectiveness in previous international trials, and the design makes the best use of the number of participating emergency departments. A stepped-wedge design is ideal for scenarios where a body of evidence for the intervention exists, yet a controlled trial evaluation is still required. ${ }^{22}$ In this study, the model strikes a balance between the researcher's need for a randomised trial to demonstrate evidence of effectiveness, and the pragmatic and clinical requirements of service partners who are responsible for providing evidence-based practice.

Randomisation of the clusters was conducted using a random number sequence by a biostatistician not involved in the project. Clusters were determined according to geographic location, with EDs located closely to each other grouped within the same cluster to minimise the risk of contamination of effect between clusters.

Outcomes are measured in each ED at every time period, hence measurement of outcomes takes place at each step in the wedge; each ED provides data points in the control and intervention conditions allowing each ED to act as its own control. ${ }^{23}$

\section{Setting and participants}

The nine EDs that have committed to participate are located in Melbourne, Sydney, Canberra and two are located in regional centres Geelong and Warrnambool, both in Victoria. In the 2013/14 financial year participating EDs had a combined total of 490000 attendances.

Patient inclusion and exclusion criteria. Inclusion criteria are all attendances by patients aged $\geq 18$ years, and being sufficiently alert and oriented to respond to study items either at triage or follow up. Exclusion criteria are patients aged $<18$, those suffering from a severe or life-threatening injury or illness, a serious mental health problem, being too intoxicated to respond to clinician questions, or being excluded at the discretion of the administering clinician. As such, in addition to Yes and No, clinicians have the following response categories available for 


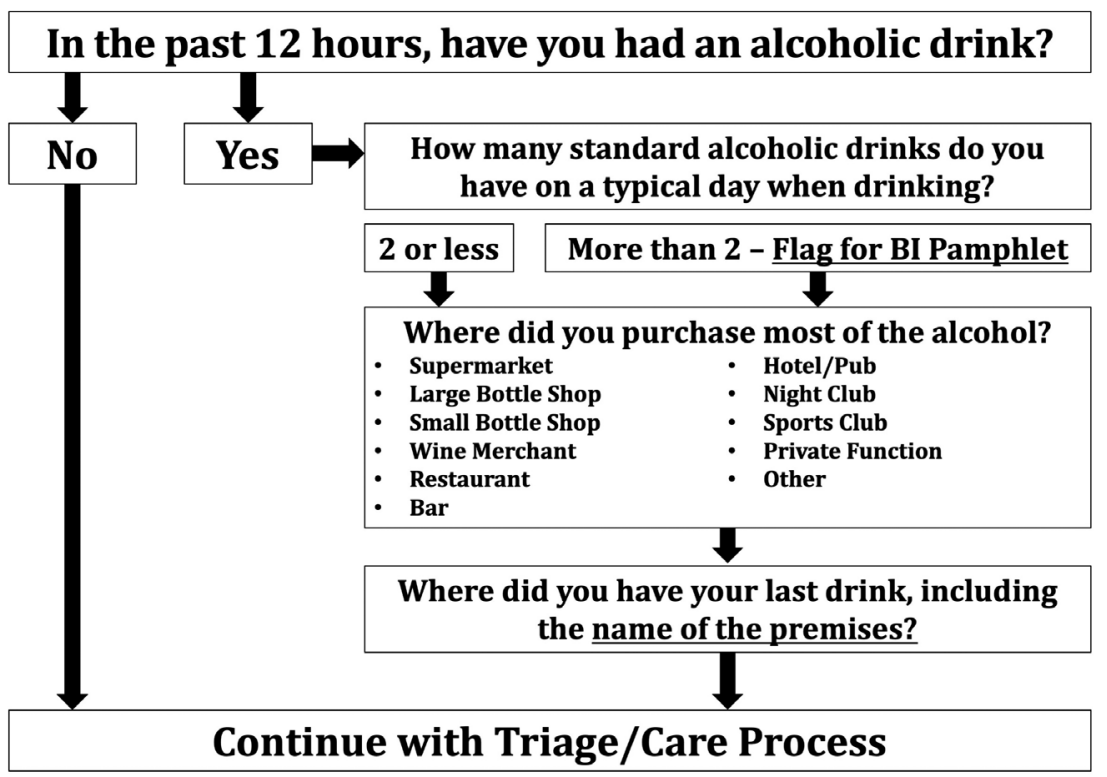

Figure 2. Mandatory data collection model.

question one of the data collection model: Non-Communicative: Patient is unconscious, too intoxicated or impaired to respond; Refused: Patient refuses to answer the question; Unknown: Patient is confused, or offers an unintelligible response; Ethical: Clinician perceives a cultural or interpersonal issue, or other clinical judgement, that makes the questions inappropriate; NESP: patient is of non-English speaking background.

\section{Data collection model}

The key principle of the last-drinks intervention model is that information is collected systematically and mandatorily, and that this information is used as intelligence guiding public health level interventions. All patients presenting to the ED who meet inclusion criteria are screened for alcohol intake. Every ED will implement systematic data collection and respond with a brief information pamphlet for every patient who screens positive for hazardous alcohol use. Implementation at each site requires modification of existing ED information systems to create a mandatory and reliable system that will have minimal impact to established clinical process (Fig. 2).

Study data (Fig. 2) are prospectively collected by ED triage nurses, clerks, and clinicians at all nine EDs as a part of standard clinical process continuously throughout the data collection period of 36 months. During the 36 month data collection period, research staff employed by the participating hospitals will extract and deidentify the last drinks data. These de-identified data will be provided to the research team for analysis and preparation of intervention materials. Starting at 9 months and subsequently at each 3 month interval, clusters of EDs will commence delivering the public health intervention according to the randomisation schedule.

\section{Measures}

Outcome measures. Primary outcomes will be: (i) both the absolute number and proportion of presentations (per total number of presentations within inclusion criteria) in which the patient reports alcohol use via the proposed data collection system; and (ii) both the number and proportion of presentations (per total number of presentations within inclusion criteria) assigned an injury diagnosis that also occurred during $\mathrm{HAH}$ (20.00 hours Friday to 06.00 hours Saturday and 20.00 hours Saturday to 06.00 hours Sunday, recorded at time of triage). This data coding method has been used in a number of previous studies. ${ }^{24-26}$

Process measures. Previous research by Boyle et al. ${ }^{12}$ found that staff acceptance of the data collection model was high when combined with feedback of project results. Process measures will monitor the logistical and feasibility concerns, barriers, challenges, and clinical impacts of implementing the intervention model in an Australian context. A sample of 80 clinical staff at participating EDs will be sought for anonymous, self-completed staff experience surveys at 1,6 , 12,24 and 36 month intervals following the commencement of data collection. Staff surveys will address estimated completion time, experience of negative feedback or negative patient reactions, reasons for avoiding or skipping study items, and perceptions of the value of the model overall. Further data will be collected regarding added time to clinical interactions and administration process. Staff feedback will be provided to all ED site managers via bimonthly newsletters to be distributed to ED staff.

Economic evaluation. The economic evaluation will use tools of economic appraisal to estimate the return on investment associated with the intervention. ${ }^{27}$ The analysis requires the development of a logic pathway to describe the aims of a research project, the activities undertaken and the subsequent research outcomes. The logic pathway will use the available evidence to estimate the likelihood that the research outcomes will be adopted by the enduser (i.e. clinicians, policy makers, police). Programme costs including labour and non-labour costs will be monitored and collected throughout the intervention period. A comparison of the economic benefits (observed changes in the primary outcomes): (i) number of presentations (per total number of presentations within inclusion criteria) in which the patient reports alcohol use via the proposed data collection system; and (ii) both the number of presentations (per total number of presentations within inclusion criteria) assigned an injury diagnosis 
that also occurred during $\mathrm{HAH}$ (20.00 hours Friday to 06.00 hours Saturday and 20.00 hours Saturday to 06.00 hours Sunday), with the economic costs of the programme, provides an indication of the return on investment, or the cost-benefit of the programme. Sensitivity analysis will be conducted to test the robustness of results where parameter uncertainty may exist.

Analysis plan and power calculations. Based on previous data the expected proportion of presentations that are alcohol related is $11 \%$ and the proportion during $\mathrm{HAH}$ is $36 \%$. A 6 month pilot trial of the data collection returned a data compliance rate of $100 \%$, including $3.1 \%$ of patients who were recorded as declining a response or non-responsive. ${ }^{14}$ Power and sample size calculations were undertaken using Stata's 'steppedwedge' programme. ${ }^{28}$ Each $\mathrm{ED}$ is expected from previous data to contribute an average of 26000 total attendances over a 6 month period of the study (total of 36 months). Using an average cluster size of 26000 and an intra-class correlation of 0.05 , with $80 \%$ power and a $5 \%$ level of significance, a sample size of 1404000 total attendances would be large enough to detect a difference between intervention and control proportions of alcohol-related attendances of $0.3 \%$ (i.e. $11-10.7 \%$ ). This would allow for a relative risk reduction for alcoholrelated injuries of $3 \%$ $(11-10.7 \% / 11 \%)$ to be detected.

Generalised linear mixed models will be used to model all outcome measures. These models will have a fixed effect for time to adjust for any temporal variation in the outcome and an indicator variable to estimate the effect of the intervention. The models will also include random effects for the variation between clusters and the variation between time within a cluster. These will account for the repeated measures on clusters over the duration of the study with the potential for seasonal effects.

\section{Conclusions}

The current approach to monitoring the burden associated with alcohol and other drug use in Australian EDs is flawed. There is no systematic method of screening and monitoring the alcohol (or drug)-related harm for patients attending the ED, nor is there any method of identifying the sources of these harms in the community. The proposed study will provide an evaluation of an innovative approach to reducing alcoholrelated harm in Australia.

\section{Acknowledgements}

The authors thank ED clinicians and administrative staff at all participating hospitals who have agreed to support this project.

The trial described in this protocol has been funded by the National Health and Medical Research Council and St Vincent's Health Australia under the Partnership Project scheme (APP1113693).

\section{Author contributions}

All authors made substantial contributions to the conception and design of the study, drafting/revisions of this manuscript and final approval of the version to be published.

\section{Competing interests}

PM has acted as a paid expert witness in legal proceedings on behalf of a licensed venue and a security firm.

\section{References}

1. Holder Y, Peden M, Krug E, Lund J, Gururaj G, Kobusingye O. Injury surveillance guidelines. Geneva: World Health Organization, 2001.

2. World Health Organization. Violence and injury prevention. Geneva: World Health Organization. [Cited 21 Nov 2013.] Available from URL: http://www.who.int/violence_injury_ prevention/violence/en/

3. Warburton AL, Shepherd JP. Development, utilisation, and importance of accident and emergency department derived assault data in violence management. Emerg. Med. J. 2004; 21: 473-7.
4. Droste N, Miller P, Baker T. Review article: emergency department data sharing to reduce alcohol-related violence: a systematic review of the feasibility and effectiveness of community-level interventions. Emerg. Med. Australas. 2014; 26: 326-35.

5. Quigg Z, Hughes K, Bellis MA. Data sharing for prevention: a case study in the development of a comprehensive emergency department injury surveillance system and its use in preventing violence and alcohol-related harms. Injury Prevent. 2012; 18: 315-20.

6. Moore SC, Brennan I, Murphy S. Predicting and measuring premises-level harm in the night-time economy. Alcohol Alcohol. 2011; 46: 357-63.

7. Benger J, Carter R. Could interagency working reduce emergency department attendances due to alcohol consumption? Emerg. Med. J. 2008; 25: 331-4.

8. Florence C, Shepherd J, Brennan I, Simon T. Effectiveness of anonymised information sharing and use in health service, police, and local government partnership for preventing violence related injury: experimental study and time series analysis. BMJ 2011; 342: d3313.

9. Bellis MA, Leckenby N, Hughes K, Luke C, Wyke S, Quigg Z. Nighttime assaults: using a national emergency department monitoring system to predict occurrence, target prevention and plan services. BMC Public Health 2012; 12: 746.

10. Pointing S, Hayes-Jonkers C, Stone R, Brinn D, Clough A. Is it worth emergency departments recording information about alcohol-related assault occurring in inner-city, late-night entertainment precincts? Emerg. Med. Australas. 2011; 23: 106-7.

11. Warburton AL, Shepherd JP. Tackling alcohol related violence in city centres: effect of emergency medicine and police intervention. Emerg. Med. J. 2006; 23: 12-7.

12. Boyle AA, Snelling K, White L, Ariel B, Ashelford L. External validation of the Cardiff model of information sharing to reduce community violence: natural experiment. Emerg. Med. J. 2013; 30: 1020-3. 
13. Florence C, Shepherd J, Brennan I, Simon TR. An economic evaluation of anonymised information sharing in a partnership between health services, police and local government for preventing violence-related injury. Inj. Prev. 2014; 20: 108-14.

14. Miller P, Droste N, Baker T, Gervis C. Last drinks: a study of rural emergency department data collection to identify and target community alcohol-related violence. Emerg. Med. Australas. 2015; 27: 225-31.

15. Drummond C, Deluca P, Coulton S et al. The effectiveness of alcohol screening and brief intervention in emergency departments: a multicentre pragmatic cluster randomized controlled trial. PLoS One 2014; 9 : e99463.

16. Mdege ND, Fayter D, Watson JM, Stirk L, Sowden A, Godfrey C. Interventions for reducing alcohol consumption among general hospital inpatient heavy alcohol users: a systematic review. Drug Alcohol Depend. 2013; 131: 1-22.

17. Havard A, Shakeshaft A, SansonFisher R. Systematic review and meta-analyses of strategies targeting alcohol problems in emergency departments: interventions reduce alcohol-related injuries. Addiction 2008; 103: 368-76.

18. National Health and Medical Research Council. Australian Guidelines to Reduce Health Risks from Drinking Alcohol. Canberra: Commonwealth of Australia, 2009.

19. Hussey MA, Hughes JP. Design and analysis of stepped wedge cluster randomized trials. Contemp. Clin. Trials 2007; 28: 182-91.

20. Brown C, Lilford R. The stepped wedge trial design: a systematic review. BMC Med. Res. Methodol. 2006; 6: 54.

21. Copas AJ, Lewis JJ, Thompson JA, Davey C, Baio G, Hargreaves JR. Designing a stepped wedge trial: three main designs, carry-over effects and randomisation approaches. Trials 2015; 16: 352.

22. Hemming $\mathrm{K}$, Haines TP, Chilton PJ, Girling AJ, Lilford RJ. The stepped wedge cluster randomised trial: rationale, design, analysis, and reporting. BMJ 2015; 350: h391.

23. Hill A-M, Waldron N, EthertonBeer C et al. A stepped-wedge cluster randomised controlled trial for evaluating rates of falls among inpatients in aged care rehabilitation units receiving tailored multimedia education in addition to usual care: a trial protocol. BMJ Open 2014; 4: e004195.

24. Miller P, Coomber K, Sonderlund A, McKenzie S. The long-term effect of lockouts on alcohol-related emergency department attendances within Ballarat, Australia. Drug Alcohol Rev. 2012; 31: 370-6.

25. Miller PG, Tindall J, Sonderlund A et al. Dealing with Alcohol and the Night-Time Economy (DANTE): Final Report. Geelong, VIC: Deakin University and Hunter New England Population Health for the National Drug Law Enforcement Research Fund, 2011.

26. Miller PG, Sonderlund A, Coomber $\mathrm{K}$ et al. The effect of community interventions targeting licensed venues on all alcoholrelated emergency department attendances. Drug Alcohol Rev. 2011; 30: 546-53.

27. Drummond MF, Stoddart GL, Torrance GW. Methods for the Economic Evaluation of Health Care Programmes. Oxford: Oxford Medical Publications, 1988.

28. Hemming K, Girling A. A menudriven facility for power and detectable-difference calculations in stepped-wedge cluster-randomized trials. Stata J. 2014; 14: 363-80. 